EUROPEAN ORGANIZATION FOR NUCLEAR RESEARCH

CERN - AB Department

CERN-AB-2007-071

CLIC Note 723

\title{
BEAM DYNAMICS STUDIES IN THE CLIC INJECTOR LINAC
}

A. Ferrari ${ }^{1)}$, A. Latina, L. Rinolfi, F. Tecker

CERN, Geneva, Switzerland

\begin{abstract}
The CLIC Injector Linac has to accelerate both electron and positron main beams from $200 \mathrm{MeV}$ up to 2.42 $\mathrm{GeV}$ prior to their injection into the pre-damping rings. Its 26 accelerating structures operate at $1.5 \mathrm{GHz}$, with a loaded gradient of $17 \mathrm{MV} / \mathrm{m}$. A FODO lattice that wraps the accelerating structures at the beginning of the linac, followed by a succession of triplet lattices between the accelerating structures, is proposed. The large normalized transverse emittance $(9200 \mathrm{~mm} . \mathrm{mrad} \mathrm{rms})$, bunch length (5mmrms) and energy spread (7 $\mathrm{MeV}$ rms) of the e+ beam set constraints on the linac, in order to reach acceptable characteristics at 2.42 $\mathrm{GeV}$ for the injection into the predamping ring. The use of a bunch compressor at the linac entrance is an option in order to achieve good performance in both the longitudinal and transverse phase spaces. Tracking studies of both electron and positron beams in the linac have been performed and are presented.
\end{abstract}

\footnotetext{
1). Uppsala University, Sweden
}

Presented at

PAC07, 22nd PAC Conference, June 25-29, 2007, Albuquerque, USA

Geneva, Switzerland

August 2007 


\title{
BEAM DYNAMICS STUDIES IN THE CLIC INJECTOR LINAC
}

\author{
A. Latina, L. Rinolfi, F. Tecker, CERN, Geneva, Switzerland \\ A. Ferrari*, Uppsala University, Sweden
}

\section{Abstract}

The CLIC Injector Linac has to accelerate both electron and positron main beams from $200 \mathrm{MeV}$ up to $2.42 \mathrm{GeV}$ prior to their injection into the pre-damping rings. Its 26 accelerating structures operate at $1.5 \mathrm{GHz}$, with a loaded gradient of $17 \mathrm{MV} / \mathrm{m}$. A FODO lattice that wraps the accelerating structures at the beginning of the linac, followed by a succession of triplet lattices between the accelerating structures, is proposed. The large normalized transverse emittance ( $9200 \mathrm{~mm} . \mathrm{mrad} \mathrm{rms})$, bunch length ( $5 \mathrm{~mm} \mathrm{rms}$ ) and energy spread ( $7 \mathrm{MeV} \mathrm{rms)} \mathrm{of} \mathrm{the} e^{+}$beam set constraints on the linac, in order to reach acceptable characteristics at $2.42 \mathrm{GeV}$ for the injection into the predamping ring. The use of a bunch compressor at the linac entrance is an option in order to achieve good performance in both the longitudinal and transverse phase spaces. Tracking studies of both electron and positron beams in the linac have been performed and are presented.

\section{INTRODUCTION}

The Compact Linear Collider (CLIC) study explores the feasibility of the Two-Beam Acceleration technology, with high-frequency traveling-wave structures at room temperature $[1,2]$. The CLIC frequency was recently changed from 30 to $12 \mathrm{GHz}$. The $e^{-}$and $e^{+}$main beams are produced in one common injection complex [3]. Downstream of the sources, one single Injector Linac accelerates alternately the $e^{-}$and $e^{+}$beams from $200 \mathrm{MeV}$ to $2.42 \mathrm{GeV}$. Then, a bending magnet separates the electrons and the positrons before they are injected into their respective damping rings, see Figure 1. These should maintain a high polarization. Therefore, the energy in the rings is constrained to certain discrete values (2.424 GeV in our case), such that the spin tune is a half-integer, in order to stay away from the strong integer spin resonance.

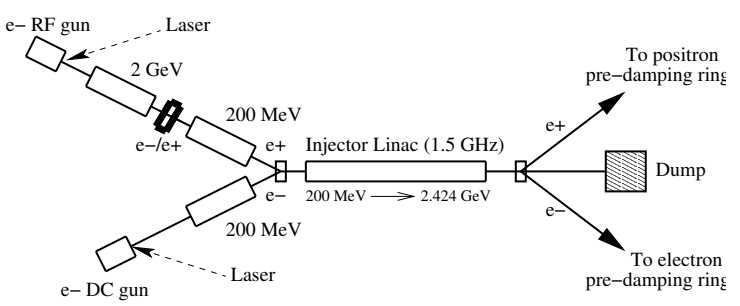

Figure 1: Schematic layout of the CLIC injector complex upstream of the pre-damping rings.

\footnotetext{
*ferrari@tsl.uu.se
}

In this paper, a design study of the CLIC Injector Linac is presented, with special emphasis on the positron main beam, which has large transverse emittances. The proposed optics is based on a FODO lattice wrapping the first six accelerating structures, followed by a succession of quadrupole triplets and accelerating structures for the rest of the linac [4]. All 26 structures operate at $1.5 \mathrm{GHz}$, they have a length of $5 \mathrm{~m}$ and a loaded gradient of $17 \mathrm{MV} / \mathrm{m}$. Particle tracking studies are performed in order to estimate the emittance growth in the linac and to determine the main beam properties before injection into the pre-damping ring.

\section{LAYOUT AND DESIGN OPTICS OF THE CLIC INJECTOR LINAC}

The Injector Linac alternately accelerates electrons and positrons. However, the most stringent constraints for its design are set by the $e^{+}$beam, because its transverse emittance is expected to be two orders of magnitude larger than for the $e^{-}$beam. Therefore, this paper mostly focuses on the beam dynamics for positrons. The main properties of the beam at the exit of the $e^{+}$Pre-Injector Linac are given in Table 1.

Table 1: Beam characteristics at the exit of the $e^{+}$PreInjector Linac [5].

\begin{tabular}{|l|c|}
\hline Beam energy $E$ & $200 \mathrm{MeV}$ \\
Normalized emittance $\gamma \epsilon_{x / y}$ & $9.2 \cdot 10^{-3} \mathrm{~m} \cdot \mathrm{rad}$ \\
Energy spread $\sigma_{E}$ & $7 \mathrm{MeV}$ \\
Bunch length $\sigma_{l}$ & $5 \mathrm{~mm}$ \\
Bunch charge $Q$ & $1.12 \mathrm{nC}$ \\
\hline
\end{tabular}

A major constraint for the design of the CLIC Injector Linac is to keep the transverse size of the positron beam as small as possible, especially in the accelerating structures. For this purpose, a FODO lattice is proposed for the six accelerating structures at the beginning of the linac. Six quadrupoles are used for each acceleration structure. Also, there is one doublet between each accelerating structure, where beam instrumentation could be inserted. For the rest of the linac, downstream of the sixth accelerating structure, another type of lattice is implemented, i.e. a succession of triplets and accelerating structures. There are four subsections, which consist of five accelerating structures with a quadrupole triplet between each of them. All these subsections start with four independent quadrupoles used both for matching the optics and for measuring the transverse properties of the beam. 
The gradients, lengths and spacings of all quadrupoles, as well as the main characteristics of the accelerating structures, are given in [4].

The betatron functions computed with the design optics along the whole CLIC Injector Linac are shown in Figure 2. At the entrance of the linac, $\beta_{x}=0.83 \mathrm{~m}$, $\alpha_{x}=-0.99$ and $\beta_{y}=2.21 \mathrm{~m}, \alpha_{y}=+2.07$. At its exit (just downstream of the last accelerating structure), one has $\beta_{x}=\beta_{y}=6.74 \mathrm{~m}$ and $\alpha_{x}=\alpha_{y}=-0.59$.

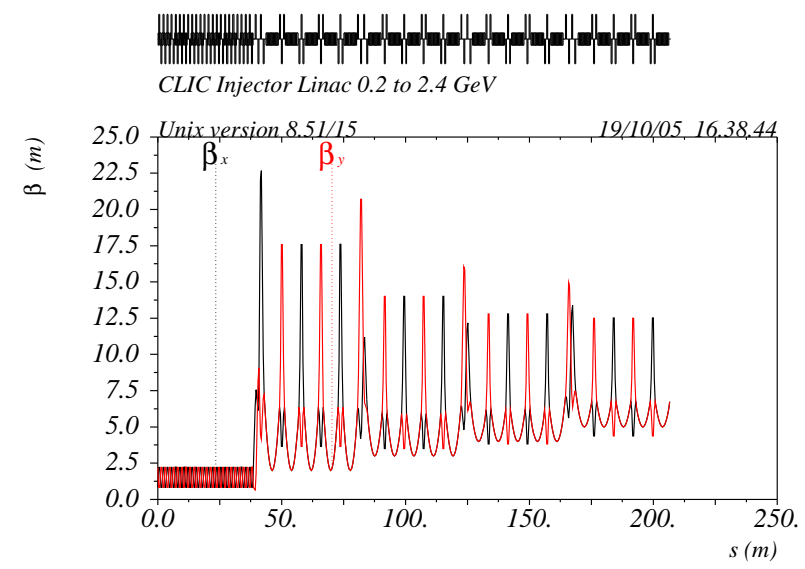

Figure 2: Betatron functions for the $e^{+}$beam (the largest values of $\beta_{x}$ and $\beta_{y}$ are obtained in the quadrupoles).

A quadrupole which is horizontally focusing for the positrons is also vertically focusing for the electrons, therefore the horizontal and vertical Twiss parameters of the electron beam at the entrance of the CLIC Injector Linac must fulfill $\beta_{x}\left(e^{ \pm}\right)=\beta_{y}\left(e^{\mp}\right)$ and $\alpha_{x}\left(e^{ \pm}\right)=\alpha_{y}\left(e^{\mp}\right)$. Consequently, at the exit of the last accelerating structure, $\beta_{x, y}$ and $\alpha_{x, y}$ are identical for electrons and positrons.

\section{PARTICLE TRACKING STUDIES}

Single bunch tracking studies of the positron beam were performed along the Injector Linac with MAD [6] and PLACET [7]. The latter takes into account the effect of the short range wakefields in the accelerating structures. Figure 3 shows a schematic layout of these structures, as used in the PLACET simulations. These were obtained by scaling down the $3 \mathrm{GHz}$ SICA structures [8].

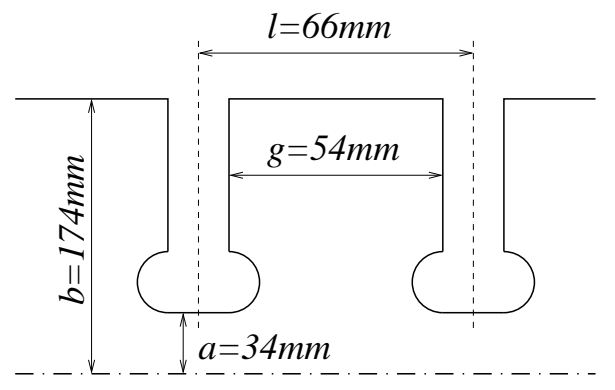

Figure 3: Schematic layout of the accelerating structures in the CLIC Injector Linac.
Gaussian distributions are used to describe the longitudinal and transverse phase spaces of the positron beam at the entrance of the linac, according to the beam properties given in Table 1. By comparing the MAD and PLACET simulation results, we conclude that the wakefields in the accelerating structures do not have a sizeable effect.

Because of the non-zero bunch length, all positrons do not receive the same amount of energy when they pass through the accelerating structures and this leads to some additional energy spread, as shown in Figure 4.

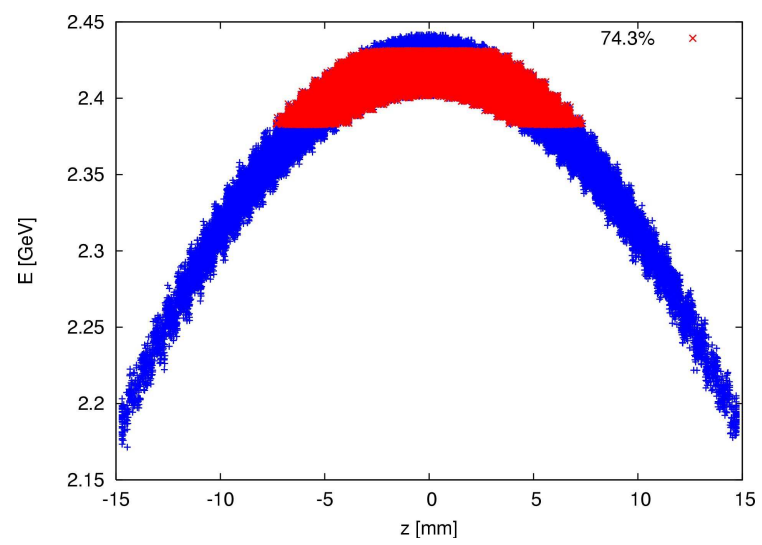

Figure 4: Longitudinal distributions of the $e^{+}$beam at the exit of the CLIC Injector Linac.

Assuming a pre-damping ring acceptance of $\pm 1 \%$ for the energy, the total number of captured positrons is $74 \%$. It corresponds to an rms bunch length of $3.2 \mathrm{~mm}$.

The larger energy spread has an impact on the transverse distributions of the positron beam at the end of the linac, due to chromatic effects. This leads to a growth of about $5 \%$ for the emittance, see Figure 5. The jump in emittance corresponds to the transition from the FODO lattice to the configuration with triplets between the accelerating structures. The Twiss parameters at the end of the linac are: $\beta_{x}=7.1 \mathrm{~m}, \alpha_{x}=-0.53$ and $\beta_{y}=6.7 \mathrm{~m}, \alpha_{y}=-0.57$.

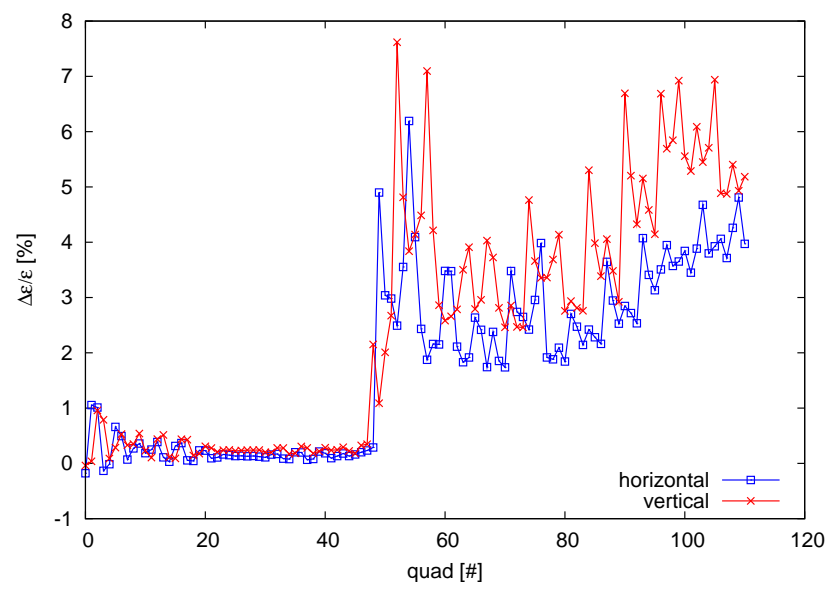

Figure 5: Transverse normalized emittances for the $e^{+}$ beam along the CLIC Injector Linac. 
The main properties of the positron beam at the end of the Injector Linac are summarized in Table 2.

Table 2: Positron beam characteristics at the exit of the Injector Linac.

\begin{tabular}{|l|c|}
\hline Beam energy $E$ & $2.42 \mathrm{GeV}$ \\
Normalized emittance $\gamma \epsilon_{x / y}$ & $9.7 \cdot 10^{-3} \mathrm{~m} . \mathrm{rad}$ \\
Energy spread $\sigma_{E}$ & $40 \mathrm{MeV}$ \\
Bunch length $\sigma_{l}$ & $5 \mathrm{~mm}$ \\
\hline
\end{tabular}

By using a bunch compressor prior to the injection of the positrons into the Injector Linac, an optimal working point could be found, in order to reduce the chromatic effects. Tracking studies with MAD and PLACET indicate that the rms energy spread can be reduced by more than a factor two, as seen in Figure 6.

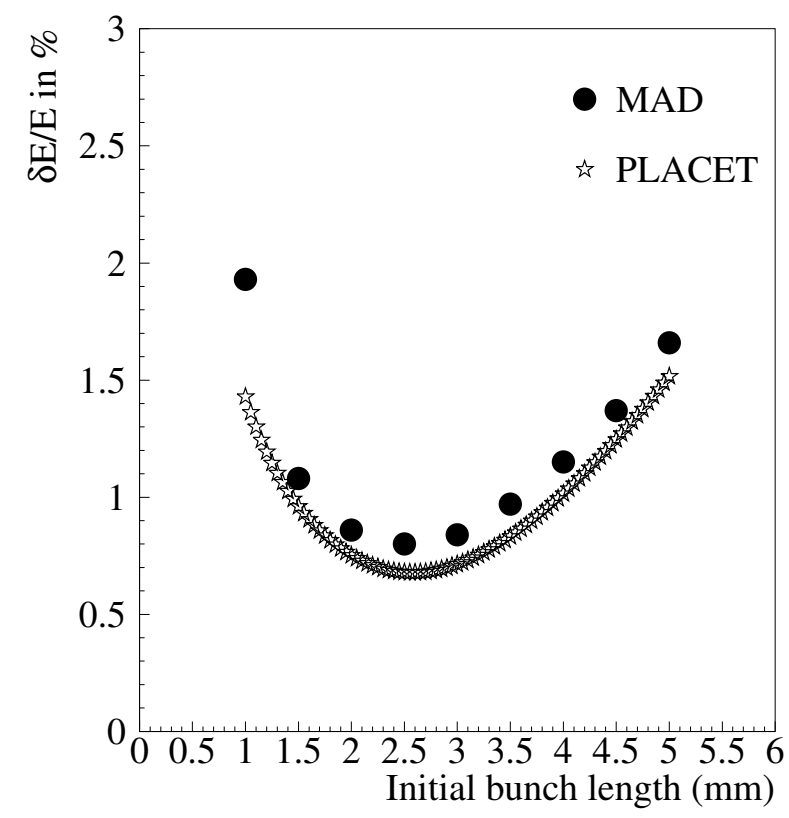

Figure 6: Energy spread (rms) at $2.42 \mathrm{GeV}$, as a function of the positron bunch length at $200 \mathrm{MeV}$.

With an initial bunch length of $2.5 \mathrm{~mm}$, the energy spread is increased to $14 \mathrm{MeV}$ (for a beam energy of $200 \mathrm{MeV}$ ) and it becomes $19 \mathrm{MeV}$ at $2.42 \mathrm{GeV}$. The bunch length remains almost unchanged, i.e. $2.7 \mathrm{~mm}$ at the end of the linac.

The normalized emittance is $9.6 \cdot 10^{-3} \mathrm{~m} . \mathrm{rad}$ in both transverse directions. As for the Twiss parameters at the exit of the last accelerating structure, we find $\beta_{x}=7.7 \mathrm{~m}$, $\alpha_{x}=-0.55$ and $\beta_{y}=6.9 \mathrm{~m}, \alpha_{y}=-0.54$.

When using the bunch compressor, the total number of positrons captured by the pre-damping ring becomes $87 \%$. It corresponds to an rms bunch length of $2.3 \mathrm{~mm}$.
The CLIC Injector Linac must also transport electrons to their pre-damping ring. The main properties of the electron beam at the beginning of the linac are given in Table 3 .

Table 3: Beam characteristics at the exit of the $e^{-}$PreInjector Linac.

\begin{tabular}{|l|c|}
\hline Beam energy $E$ & $200 \mathrm{MeV}$ \\
Normalized emittance $\gamma \epsilon_{x / y}$ & $100 \cdot 10^{-6} \mathrm{~m} . \mathrm{rad}$ \\
Energy spread $\sigma_{E}$ & $1 \mathrm{MeV}$ \\
Bunch length $\sigma_{l}$ & $1 \mathrm{~mm}$ \\
Bunch charge $Q$ & $0.75 \mathrm{nC}$ \\
\hline
\end{tabular}

For the electron beam, single bunch PLACET tracking simulations show that, at the exit of the Injector Linac, the energy spread is $1.8 \mathrm{MeV}$ and the bunch length remains unchanged. Also, no emittance growth is observed for the electron beam and the Twiss parameters derived from the tracking simulations are identical to those obtained for the positron beam.

\section{CONCLUSION}

This paper presents the results of MAD and PLACET particle tracking studies performed for the CLIC Injector Linac, which accelerates the main beams from $200 \mathrm{MeV}$ to $2.42 \mathrm{GeV}$. For both the electron and positron beams, there is practically no emittance growth. Also, with the present radial aperture of $34 \mathrm{~mm}$ for the accelerating structures and a maximum rms beam size of $7 \mathrm{~mm}$ for the positrons at the entrance of the first structure, the geometrical acceptance corresponds to $5 \sigma$.

The design of the $e^{+}$pre-damping ring is a crucial topic to address, where the six-dimensional phase-space must be accomodated in order to capture the maximum number of positrons. The design of the $e^{-}$pre-damping ring is also necessary, as an intermediate step in order to reach the CLIC emittances at the exit of the damping ring with an acceptable dynamic aperture.

\section{REFERENCES}

[1] http://clic-study.web.cern.ch/CLIC-Study/

[2] R. Corsini, "Results on CLIC Proof of Principle from CTF3", these proceedings.

[3] L. Rinolfi, "A CLIC injector complex for the main beams", CLIC note 354 .

[4] A. Ferrari, L. Rinolfi and F. Tecker, "Design study of the CLIC main beam Injector Linac", CLIC note 626.

[5] T. Kamitani and L. Rinolfi , "The CLIC positron production scheme", CLIC note 537, CERN-PS-2002-071; LINAC2002 in Gyeongju, South Korea.

[6] http://mad.home.cern.ch/mad/mad8web/mad8.html

[7] http://savannah.cern.ch/projects/placet

[8] E. Jensen, "CTF3 Drive Beam Accelerating Structure", CLIC note 538 . 